\title{
Automatic 3-D information extraction of open-cast mining infrastructure from simulated IKONOS data
}

\author{
A. Almer', C. Banninger ${ }^{1}$, J.L. Fernandez-Turiel ${ }^{2}$, and J.F. Llorens ${ }^{2}$ \\ 1 Institute for Digital Image Processing, Joanneum Research, Graz, Austria \\ ${ }^{2}$ Insitut de Ciencies de 'Jaume Almera', CSSIC, Barcelona, Spain
}

\begin{abstract}
A fully automatic approach for extracting 3-D information from simulated $1 \mathrm{~m}$ panchromatic digital stereo data was tested within the context of mapping the infrastructure of an open-cast coal mine, and the accuracy of the derived products assessed in comparison to that produced from analogue aerial photographs by standard interactive photogrammetric procedures.
\end{abstract}

Key Words: IKONOS, 3-D data, automatic information extraction, open-cast mining, infrastructure.

\section{INTRODUCTION}

The object of the study was to test a fully automatic approach for extracting 3-D information from the As Pontes open-cast coal mine situated in north-western Spain from simulated IKONOS digital stereo data generated from aerial photography, to ascertain the accuracy at which information could be extracted from by the procedure in comparison to that obtainable from analogue aerial photographs by traditional interactive photogrammetric techniques.

\section{MATERIALS AND METHODS}

Colour aerial photography (Figure 1) of the mine area at a scale of 1:22 000 was scanned and digitised to a resolution of $1 \mathrm{~m}$ panchromatic and $3 \mathrm{~m}$ multispectral, to simulate IKONOS data (Figures 2 and 3 ) of comparable resolutions. The derived digital imagery were geo-referenced, using a $10 \mathrm{~m}$ resolution digital elevation model photogrammetrically created from the aerial photography.
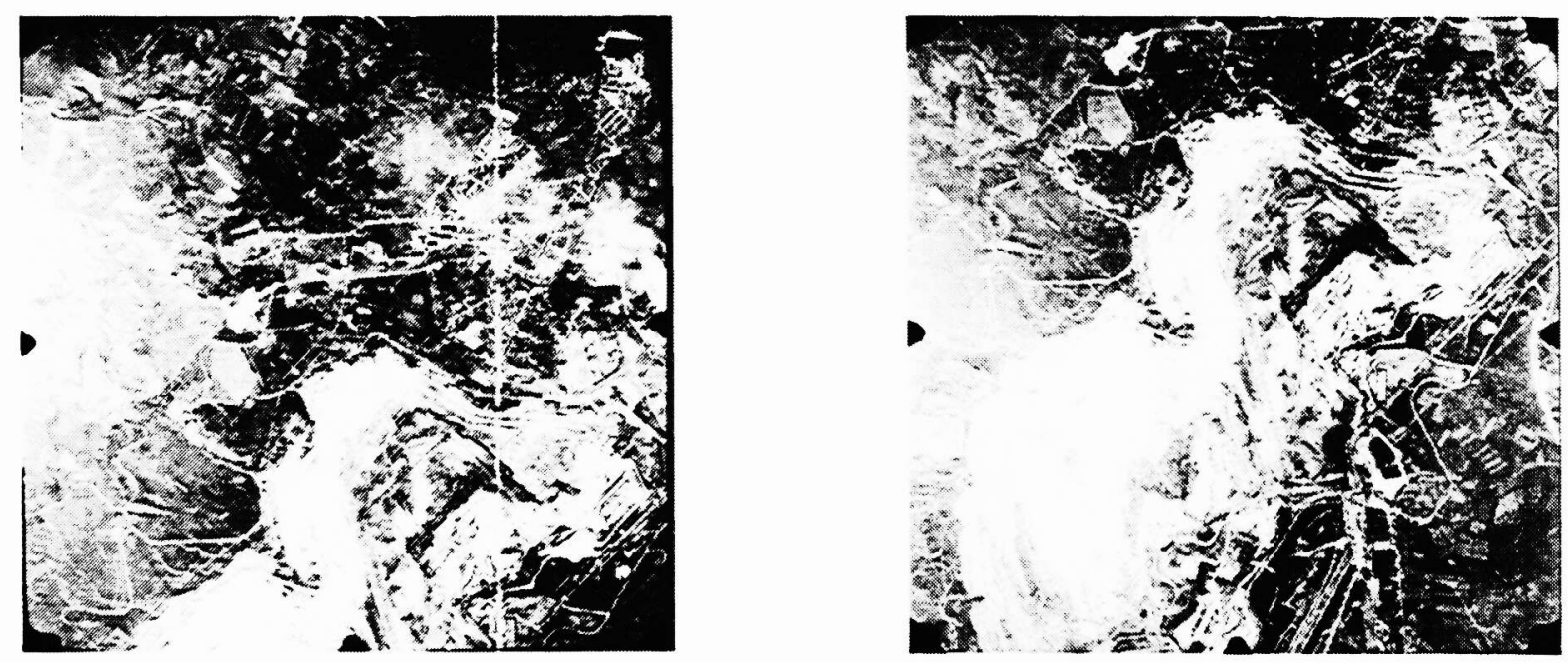

Figure 1: Colour Aerial Photography (1:22 000) of Open-Cast Coal Mine (As Pontes, Spain) 


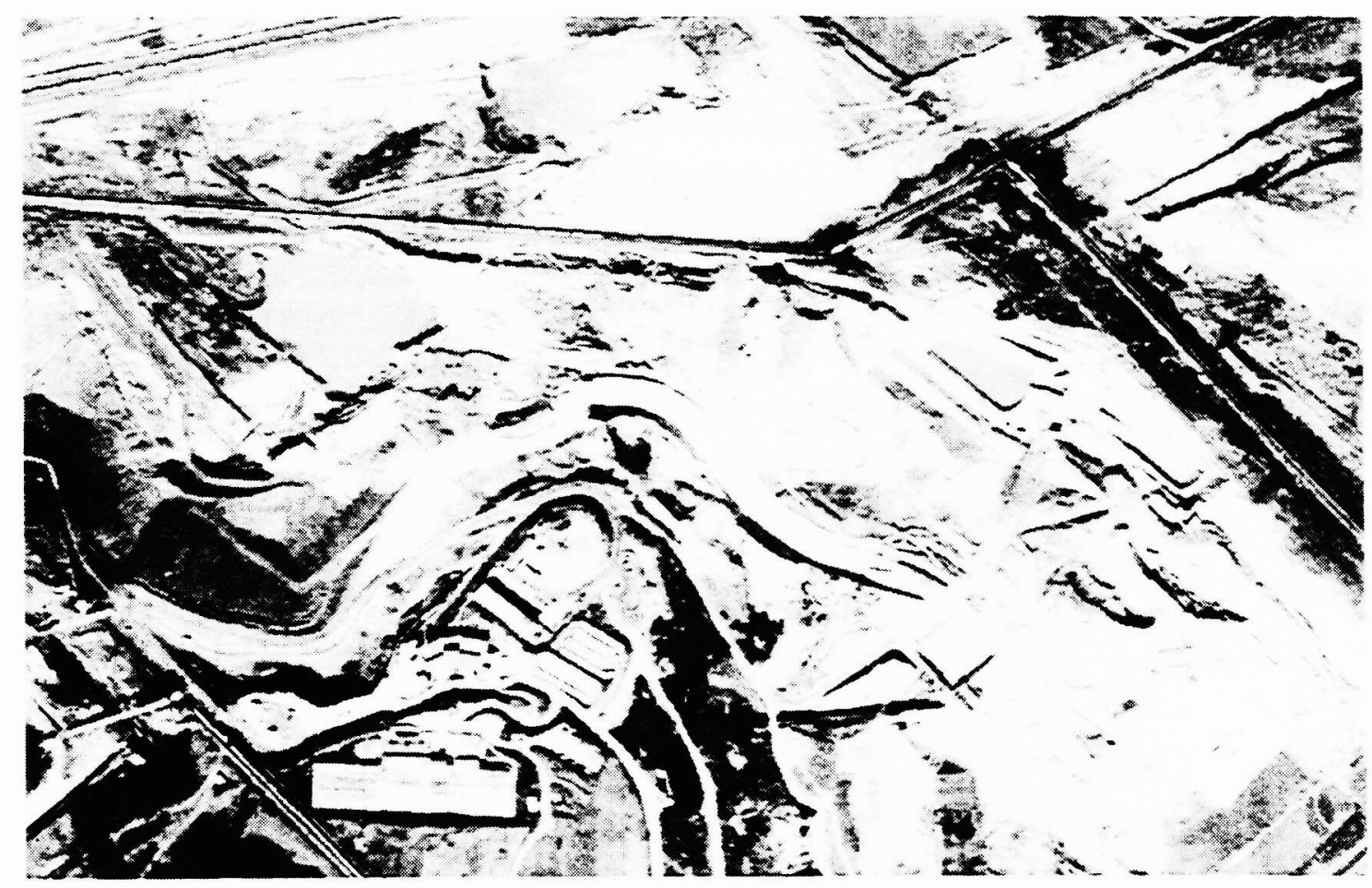

Figure 2: Simulated $1 \mathrm{~m}$ IKONOS Panchromatic Image of As Pontes Coal Mine Workings

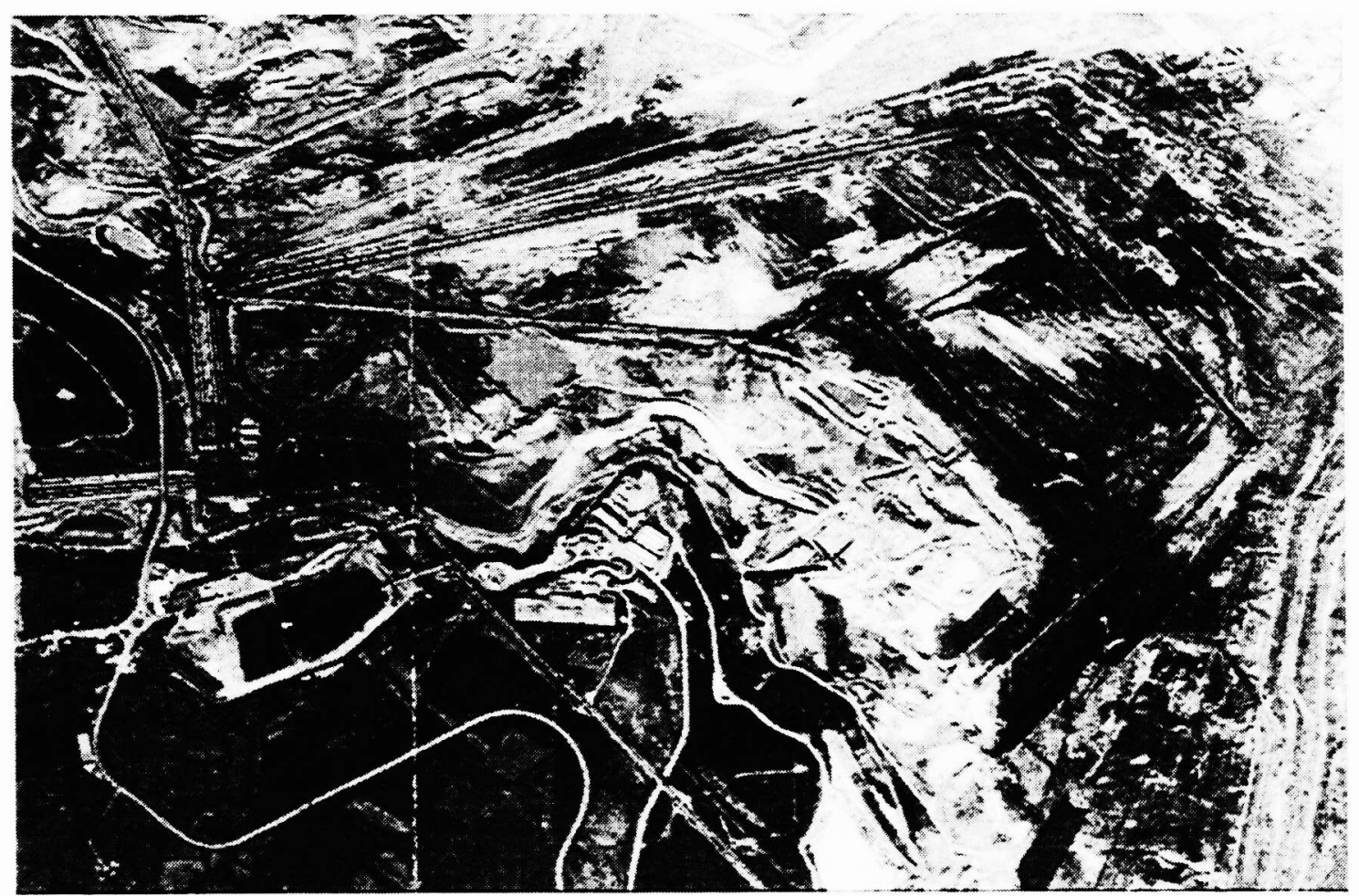

Figure 3: Simulated $3 \mathrm{~m}$ IKONOS Multispectral Image of As Pontes Coal Mine Workings

Remote sensing stereo image pairs in digital format can be used to derive three-dimensional terrain information similar to that obtainable from conventional photography. For digital data, the stereo processing chain comprises the following steps: 
1. Geometric modelling of stereo images (i.e., the determination and optimisation of parametric imaging models of the stereo data).

2. Automatic stereo image correlation (i.e., locating corresponding points in registered stereo images).

3. Generation and optimisation of a digital elevation model (DEM) from stereo correlation data (i.e., calculation of 3-D co-ordinates and interpolation of elevation raster data).

These steps are schematically displayed in Figure 4. For stereo mapping from digital data, the performance of the geometric modelling is normally limited by pixel size and stereo intersection angle.

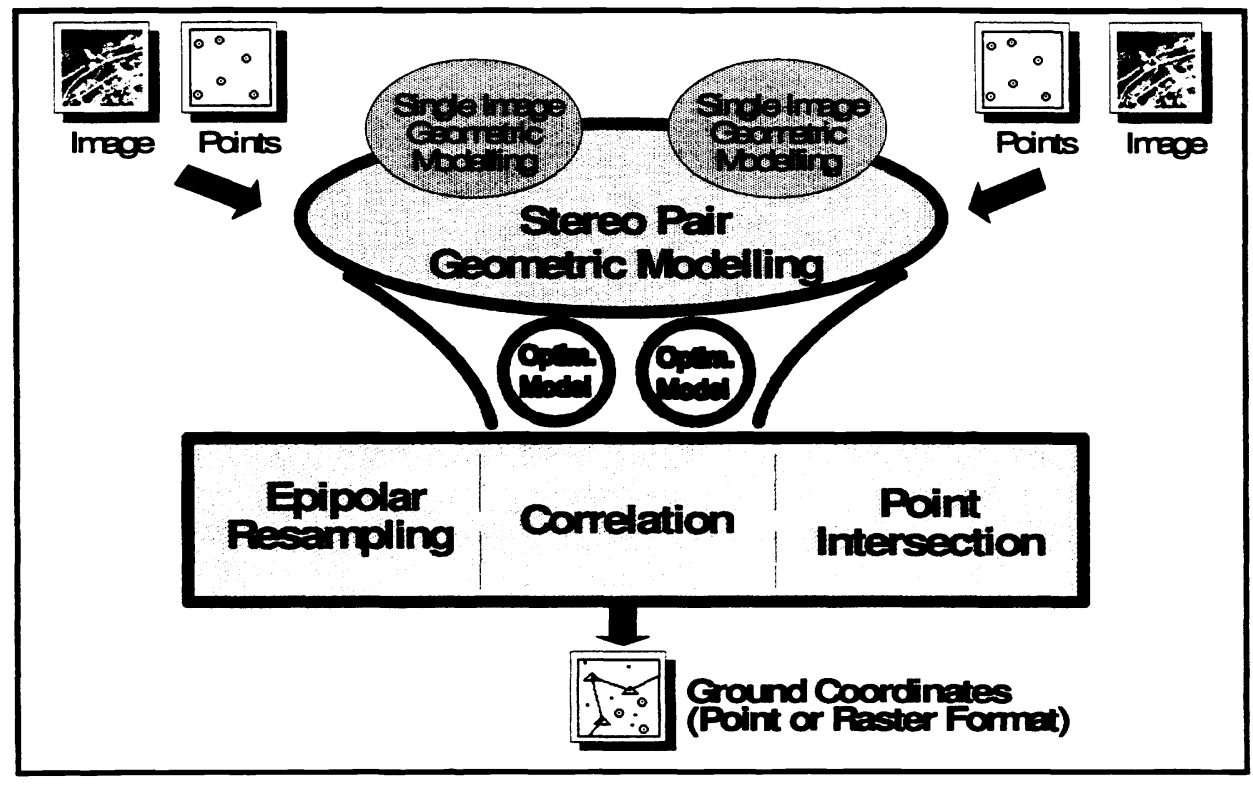

Figure 4: Schematic Representation of Stereo Mapping Procedure

Fourteen common ground control points (GCP's) on the IKONOS stereo imagery, reference DEM, and planimetric maps of the mine site and an additional 22 homologue image points were used for the stereo adjustment. The statistical parameters of the stereo adjustment are given in Table 1 and closely correspond to those obtained by the photogrammetric approach.

Table 1: Stereo Model Residual Errors

\begin{tabular}{|c|c|c|c|c|c|}
\hline GCPis $(14)$ & East $(\mathrm{m})$ & North $(\mathrm{m})$ & Height $(\mathrm{m})$ & 12DI $(\mathrm{m})$ & I3DI $(\mathrm{m})$ \\
\hline RMS & 0.5 & 0.6 & 1.1 & 0.8 & 1.4 \\
\hline MEAN & 0.0 & 0.0 & -0.3 & 0.7 & 1.3 \\
\hline STD & 0.5 & 0.6 & 1.1 & 0.4 & 0.5 \\
\hline MIN & -1.0 & -1.1 & -1.9 & 0.2 & 0.6 \\
\hline MAX & 0.8 & 1.5 & 1.8 & 1.5 & 2.3 \\
\hline
\end{tabular}




\section{RESULTS}

Based on the adjusted image parameter models and reference DEM, an orthophoto was generated and compared to reference vector data derived from the reference mine maps. The results are shown in Figure 5. The smallness of the residual errors contained in the stereo model is seen by the close correspondence between the mine infrastructure vector data and their feature representation in the orthophoto.

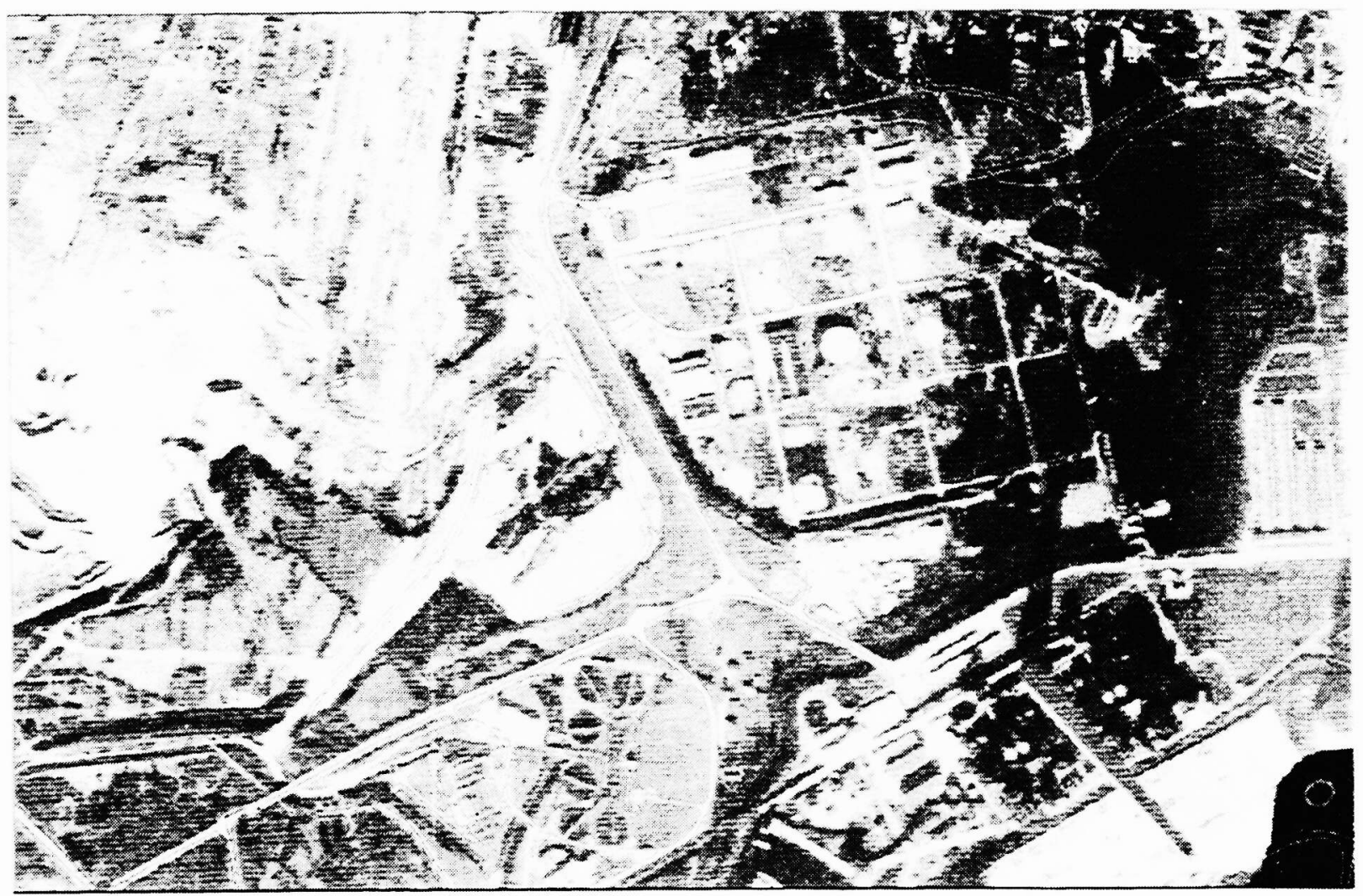

Figure 5: Aerial Orthophoto Overlain with Transport Infrastructure Vector Data

The procedure for the automatic extraction of stereo data involves three steps, which are schematically shown in Figure 6:

Step 1: Relative registration of one of the stereo image pairs to the other by epipolar resampling, using a linear pixel transformation.

Step 2: Matching of homologue points in the stereo pair, using an automatic image correlation procedure. Two different correlation algorithms were tested, the product-moment and a feature-based, both of which produced similar results.

Step 3: Transformation of two-dimensional image pixel co-ordinates in the stereo pair derived in Step 2 to threedimensional map pixel co-ordinates. A 3-D stereo intersection of geometry-specific projection lines and curves was performed and, using the derived pixel measurements, Cartesian $X, Y$, and $Z$ and map $E$ (east), $\mathrm{N}$ (north), and $\mathrm{H}$ (height) co-ordinates calculated, as shown schematically in Figure 7. 


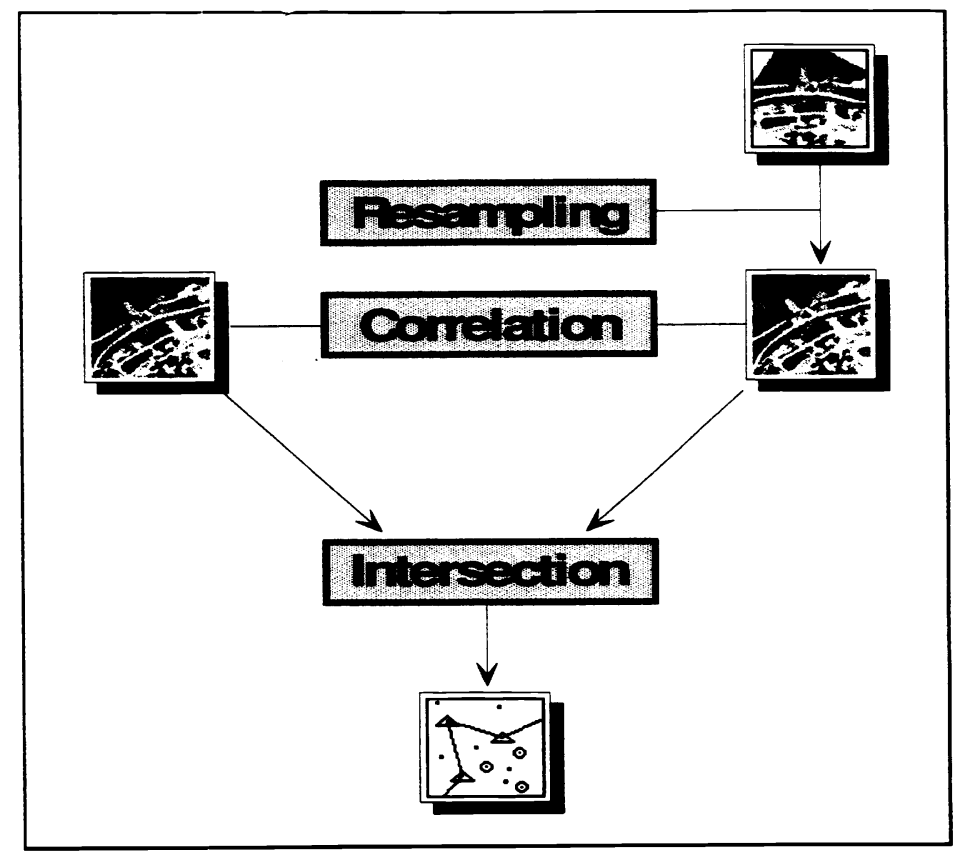

Figure 6: Schematic Presentation of Automatic Stereo Mapping Procedure
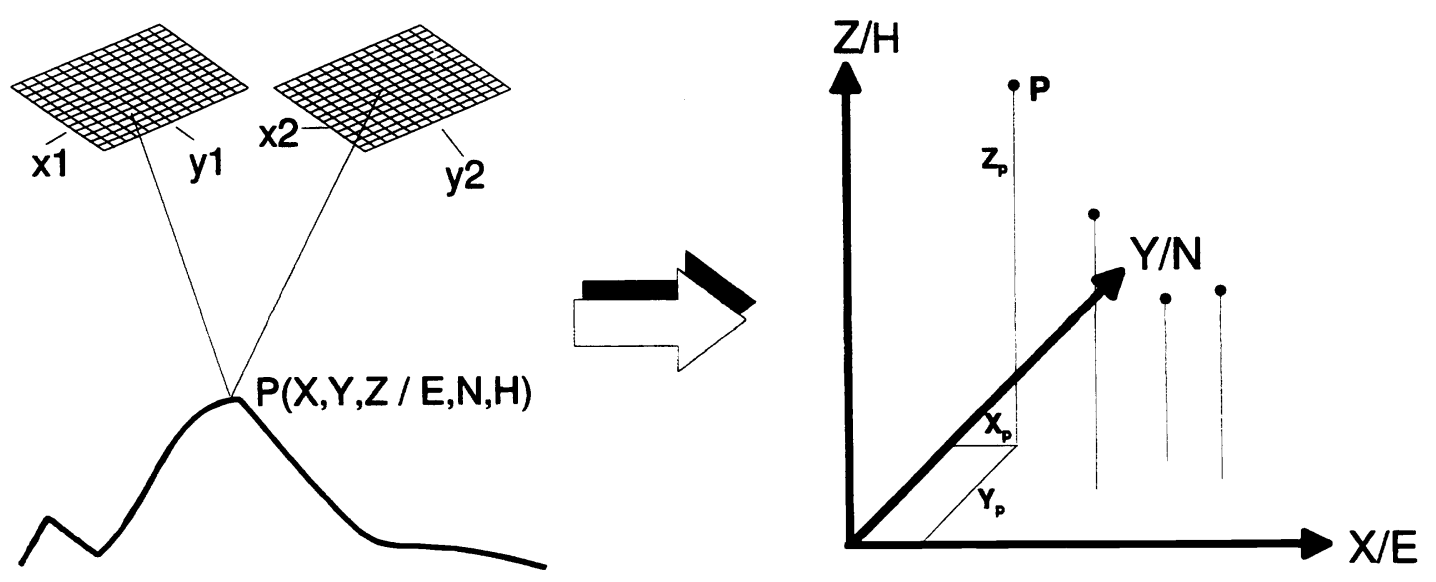

Figure 7: Point Intersection Transformation Procedure

A DEM of the mine site was generated, using the East, North, and Height co-ordinates of feature points in the IKONOS stereo image pair derived in Step 3. Because a dense set of raster points was created by the pixel-bypixel output of the stereo mapping procedure, straightforward resampling of the points was performed by direct interpolation. Figures 8 and 9 show the part of the DEM created from aerial photographs and that from IKONOS stereo imagery corresponding to the border region of the mine pit, and Table 2 gives the statistical comparison of height information derived from the two DEM's of this region. 


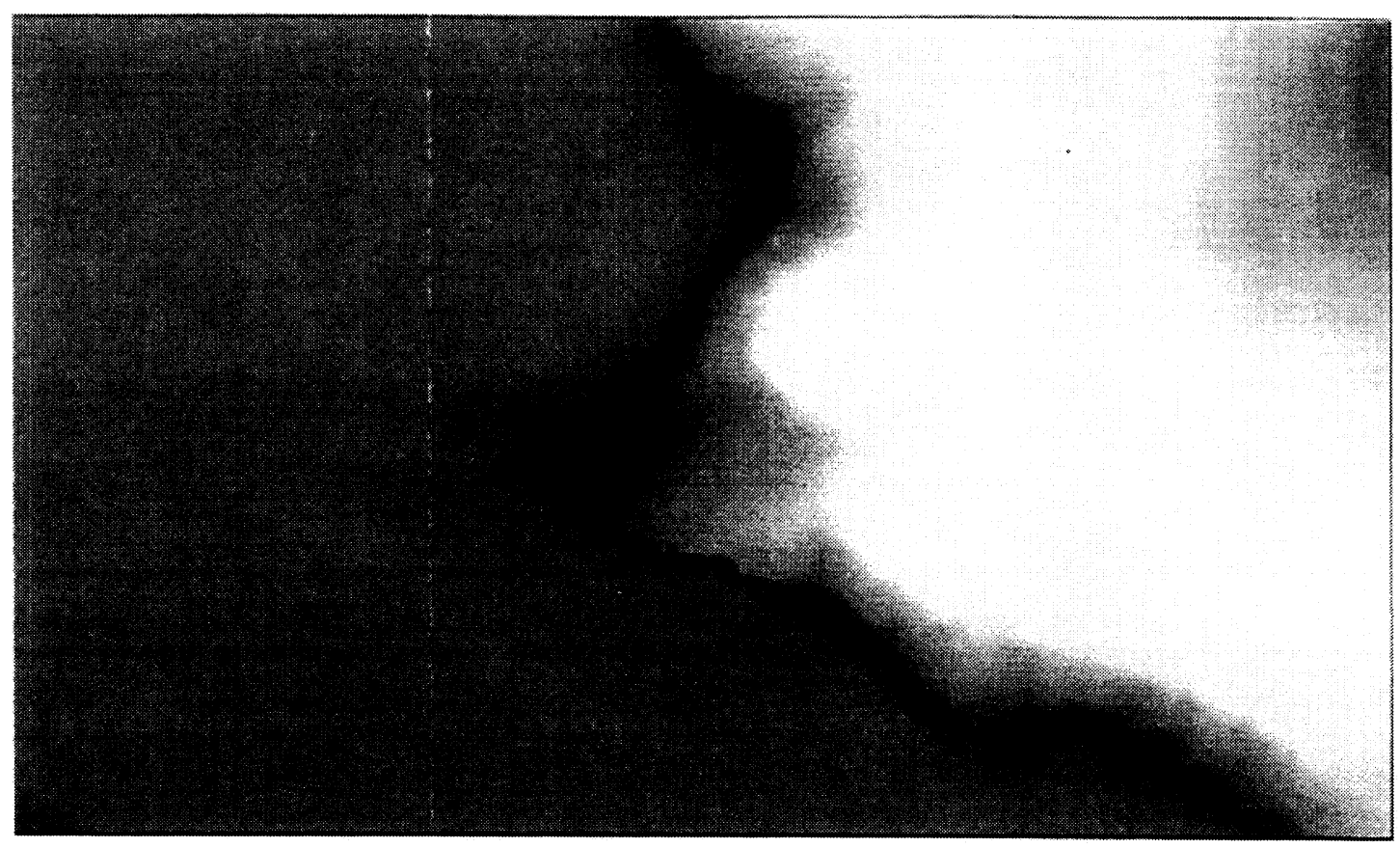

Figure 8: DEM of Part of Mine Based on Aerial Photographs

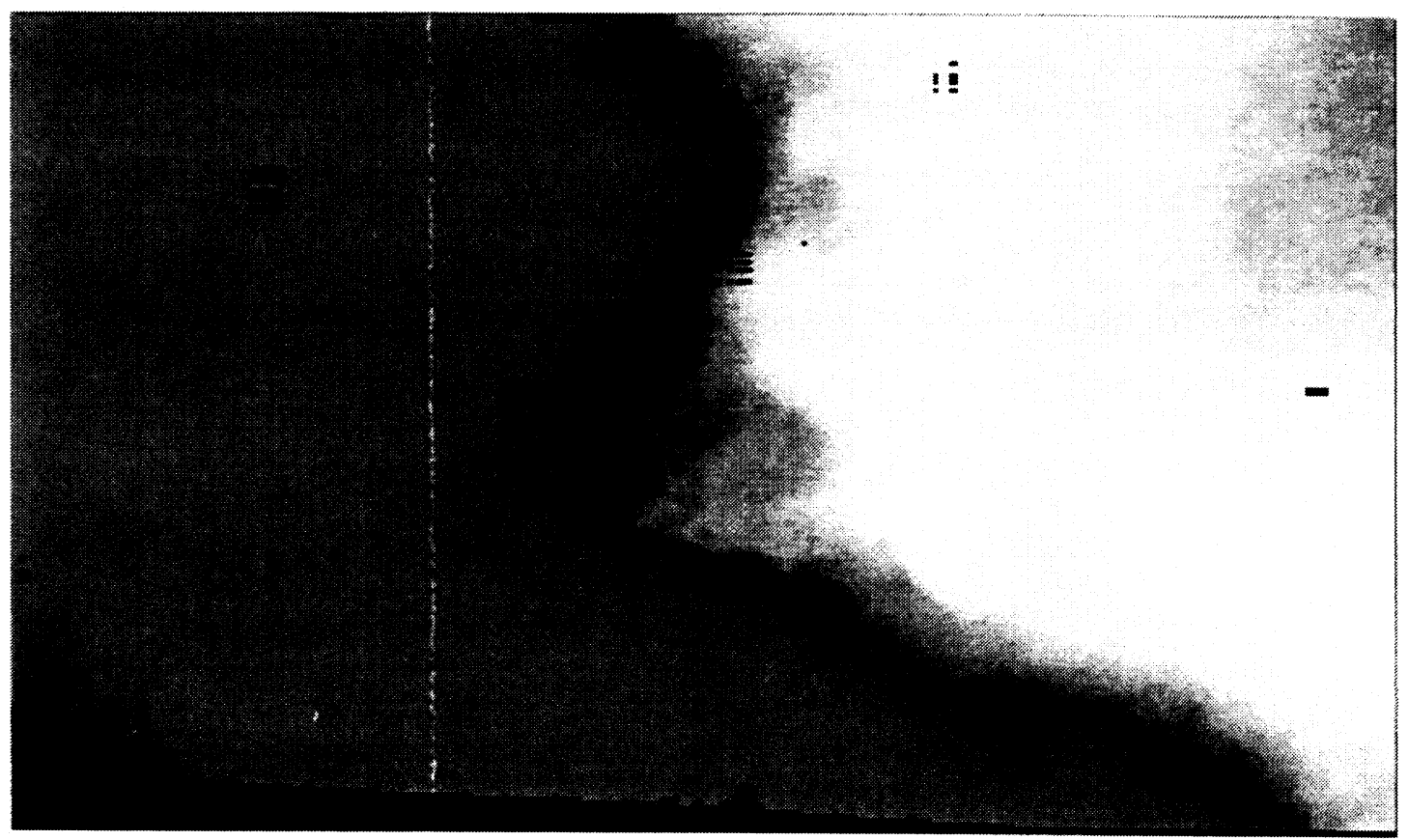

Figure 9: DEM of Part of Mine Based on IKONOS Stereo Imagery 
Table 3: DEM Comparison Statistics

\begin{tabular}{|c|c|}
\hline & $\Delta$ Height $(\mathrm{m})$ \\
\hline STD & 2.1 \\
\hline MEAN & 0.6 \\
\hline MIN & -6.9 \\
\hline MAX & 6.9 \\
\hline
\end{tabular}

\section{Discussion}

The testing of a fully automatic stereo mapping procedure to extract 3-D information from simulated $1 \mathrm{~m}$ resolution IKONOS data gave rise to a number of problems associated with the high information content of such imagery in comparison with, for example, SPOT $10 \mathrm{~m}$ panchromatic imagery. As would be expected, mine features in IKONOS imagery show much more detail than in SPOT imagery, which, for not completely understood reasons, gave rise to problems with the automatic image correlation procedure and led to a high degree of correlation failures.

An additional problem with very high resolution imagery arises from the topographic expression of the coal mine, with its steep to almost vertical pit slopes and flat, homogeneous areas surrounding the pit that result in extreme image disparities and local image differences that are difficult to cope with by automatic image correlation.

Future work will focus on optimising image correlation for high resolution imagery, such as IKONOS, by either eliminating or reducing correlation failures, and the extraction of additional information, such as spot heights and breaklines, in a semi-automatic approach. The goal is to achieved the same degree of geometric accuracy in the pit, itself, as for the pit edge by a semi-automatic procedure.

\section{ACKNOWLEDGEMENTS}

The work presented was performed as part of the ASTERISMOS Project funded by DG XII of the European Commission (contract number ENV-CT96-0373) within the $4{ }^{\text {th }}$ Framework Environment Programme. 